\title{
Auf dem Rücken der Beschäftigten - Standortwerbung in Ostdeutschland
}

\section{HINTERGRUND}

Die Ankündigung und Entscheidung Nokias, seine Mobilfunkproduktion in Deutschland (Bochum) aufzugeben und diese aus Kostengründen nach Rumänien zu verlagern, setzte die Standort- und Subventionspolitik in Deutschland jüngst massiv unter Druck. Der Nokia-Standort in Bochum wurde in der Vergangenheit mit erheblichen öffentlichen Mitteln (Investitionsbeihilfen und Forschungsförderung) subventioniert. Doch trotz Rekordgewinnen und staatlicher Förderung ist der Bochumer Standort gegenüber einer Werksneuansiedlung in Rumänien unterlegen. Der Fall Nokia zeigt einmal mehr, wie brüchig die regionale Verbundenheit von Unternehmen ist. Beschäftigte sind bei solchen Standortentscheidungen die Leidtragenden. Denn in beschäftigungspolitisch schwierigen Zeiten kann der Verlust des Arbeitsplatzes gleichbedeutend sein mit Arbeitslosigkeit.

Die derzeitige kritische Diskussion zur Standortpolitik in Deutschland vernachlässigt aber einen weiteren (für die Beschäftigten) wichtigen Punkt: Für den Standort Deutschland wird weltweit geworben, was begrüßenswert ist. Allerdings geschieht dies - vor allem in Ostdeutschland - vielfach mit zweifelhaften Argumenten. Die Inhalte der Standortwerbung machen das anschaulich.

Bei der Standortwerbung gelten niedrige Löhne, lange Arbeitszeiten und schwache Gewerkschaften als positive Faktoren, die Ostdeutschland weltweit als attraktiven Wirtschaftsstandort für Unternehmen und Investitionen auszeichnen. Vergleichbare Aussagen gibt es für Westdeutschland und insbesondere für strukturschwache westdeutsche Regionen nicht. Dies zeigen die

\begin{tabular}{|c|c|c|c|}
\hline \multicolumn{4}{|c|}{ Übersicht 1: Ministerien und Förderinstitute der Wirtschaftsförderung } \\
\hline \multirow{2}{*}{$\begin{array}{l}\text { Bund/ } \\
\text { Bundesländer }\end{array}$} & \multirow[t]{2}{*}{ Ministerien } & \multicolumn{2}{|c|}{ Nachgelagerte Institutionen } \\
\hline & & Förderbanken & Wirtschaftsfördergesellschaften \\
\hline Bund & $\begin{array}{l}\text { Bundesministerium für } \\
\text { Wirtschaft und } \\
\text { Technologie }\end{array}$ & KfW Bankengruppe & Invest in Germany GmbH \\
\hline \multirow[t]{2}{*}{ Berlin } & $\begin{array}{l}\text { Senatsverwaltung für } \\
\text { Wirtschaft, Technologie } \\
\text { und Frauen }\end{array}$ & $\begin{array}{l}\text { Investitionsbank } \\
\text { Berlin (IBB) }\end{array}$ & $\begin{array}{l}\text { Berlin Partner GmbH } \\
\text { Business Location Center }\end{array}$ \\
\hline & $\begin{array}{l}\text { Zentrale Anlauf- und } \\
\text { Koordinierungsstelle für } \\
\text { Unternehmen (ZAK) }\end{array}$ & & \\
\hline Brandenburg & $\begin{array}{l}\text { Ministerium für } \\
\text { Wirtschaft }\end{array}$ & $\begin{array}{l}\text { Investitionsbank } \\
\text { des Landes } \\
\text { Brandenburg (ILB) }\end{array}$ & $\begin{array}{l}\text { ZukunftsAgentur Brandenburg } \\
\text { (ZAB) }\end{array}$ \\
\hline $\begin{array}{l}\text { Mecklenburg- } \\
\text { Vorpommern }\end{array}$ & $\begin{array}{l}\text { Ministerium für Wirtschaft, } \\
\text { Arbeit und Tourismus }\end{array}$ & $\begin{array}{l}\text { Landesförderinstitut } \\
\text { Mecklenburg- } \\
\text { Vorpommern }\end{array}$ & $\begin{array}{l}\text { Invest in Mecklenburg- } \\
\text { Vorpommern GmbH }\end{array}$ \\
\hline Sachsen & $\begin{array}{l}\text { Staatsministerium für } \\
\text { Wirtschaft und Arbeit }\end{array}$ & $\begin{array}{l}\text { Sächsische } \\
\text { Aufbaubank - } \\
\text { Förderbank (SAB) }\end{array}$ & $\begin{array}{l}\text { Wirtschaftsförderung Sachsen } \\
\text { GmbH (WFS)/Invest in Saxony }\end{array}$ \\
\hline Sachsen-Anhalt & $\begin{array}{l}\text { Ministerium für Wirtschaft } \\
\text { und Arbeit }\end{array}$ & $\begin{array}{l}\text { Investitionsbank } \\
\text { Sachsen-Anhalt }\end{array}$ & $\begin{array}{l}\text { Investitions- und } \\
\text { Marketinggesellschaft } \\
\text { Sachsen-Anhalt mbH }\end{array}$ \\
\hline Thüringen & $\begin{array}{l}\text { Ministerium für Wirtschaft, } \\
\text { Technologie und Arbeit }\end{array}$ & $\begin{array}{l}\text { Thüringer } \\
\text { Aufbaubank (TAB) }\end{array}$ & $\begin{array}{l}\text { Landesentwicklungsgesellschaft } \\
\text { Thüringen mbH (LEG) }\end{array}$ \\
\hline $\mathrm{Q}$ & utoren. & & WSI mitTEILUNGEN \\
\hline
\end{tabular}

Internet-Seiten der werbenden Institutionen der ostdeutschen Wirtschaftsförderung, die wir untersucht haben. ${ }^{1}$ Es handelt sich dabei um das Bundesministerium für Wirtschaft und Technologie sowie die in den einzelnen Bundesländern für die Wirtschaftsförderung und damit für die Standortwerbung verantwortlichen Wirtschaftsministerien und von ihnen beauftragte Einrichtungen. In erster Linie sind hier die Landesinvestitions- und -förderbanken zu nennen, aber auch die Wirtschaftsförderungsgesellschaften. Diese sind entweder im Besitz des jeweiligen Bundeslandes bzw. des Bundes oder anteilig im Besitz vertretungsberechtigter Institutionen der privaten Wirtschaft (z. B. Industrie- und Handelskammern). Oder es handelt sich um Interessensvertretungen der privaten Wirtschaft und Banken. Ergänzt werden die ost- deutschen Wirtschaftsfördergesellschaften durch die Invest in Germany GmbH auf der Ebene des Bundes.

Übersicht 1 zeigt, welche Einrichtungen wir berücksichtigt haben. Die fett markierten Institutionen stellen in ihrer Standort-

\footnotetext{
1 Die dem Beitrag zugrunde liegenden Quellen und Zitate sind verfügbar unter http://www.boeckler. de/pdf/wsi_standortwerbung_tab_ziegler.pdf
}

Astrid Ziegler, Dr., ist Wissenschaftlerin im WSI in der Hans-Böckler-Stiftung. Arbeitsschwerpunkt: Strukturforschung und -politik e-mail: Astrid-Zieg/er@boeckler.de Marco Geis, studiert Politikwissenschaft an der Philipps-Universität Marburg und arbeitete als Praktikant im WSI. e-mail: geis.marco@web.de 
werbung auf die schlechten Arbeitsbedingungen in Ostdeutschland als positiven Standortfaktor ab. Wie unsere Analyse zeigt, ist dies nicht die Ausnahme, sondern die Regel, trifft doch die Praxis, mit schlechten Arbeitsbedingungen zu werben, für alle Wirtschaftsförderungsgesellschaften und die Hälfte der Wirtschaftsministerien zu.

\section{ANSATZPUNKTE DER STANDORT- WERBUNG}

Standortwerbung setzt an den räumlichen Standortbedingungen an. Hervorgehoben werden die Qualitäten und Stärken eines Standortes, um positiv auf Standortentscheidungen der Wirtschaft einzuwirken. Und tatsächlich wird die Entscheidung eines Unternehmens, einen bestimmten Standort als Sitz zu wählen und dort zu investieren, von einer Reihe von Faktoren beeinflusst. Kriterien wie die Anbindung an Zulieferbetriebe, an Distributionsschienen, ans Verkehrsnetz allgemein sowie gesetzliche, steuerliche und Wettbewerbsfaktoren fließen in die Entscheidung ein. Je nach konkreter Ausprägung der Standortfaktoren entscheidet sich ein Unternehmen für oder gegen einen potenziellen Standort. In der Literatur findet sich folgende Systematisierung von Standortfaktoren:

(1) Allgemeine und spezielle Standortfaktoren:

Allgemeine Faktoren sind solche, die für alle Unternehmen relevant sind. So gilt beispielsweise der kommunale Steuerhebesatz für alle Unternehmen mit Sitz in der Kommune. Spezielle Standortfaktoren sind dagegen nur für bestimmte Branchen wichtig. Das Vorhandensein eines Hafens ist z. B. vor allem für die Schwerindustrie von strategischer Bedeutung.

(2) Harte und weiche Standortfaktoren: Harte Standortfaktoren sind wirtschaftlich unmittelbar relevant, wie z. B. die Transportkosten oder das Einkommensniveau. Weichen Standortfaktoren werden außerökonomische Wirkungen zugeschrieben, wie z. B. der Freizeitwert oder die landschaftliche und kulturelle Attraktivität.

(3) Standortfaktoren räumlicher Reichweite:

Hier lassen sich im Wesentlichen drei Ebenen, je nach internationaler, nationaler oder regionaler Standortwahl, unterscheiden. Zur ersten Ebene (Land) gehören u. a.

\begin{tabular}{|c|c|}
\hline \multicolumn{2}{|c|}{$\begin{array}{l}\text { Übersicht 2: Kriterien der Standortentscheidung auf unterschiedlichen } \\
\text { räumlichen Ebenen }\end{array}$} \\
\hline Räumliche Ebene & Kriterien \\
\hline Land & $\begin{array}{l}\text { Steuern, politische und wirtschaftliche Stabilität, Gewerkschaften, Inflation, } \\
\text { Wachstum, Bundesförderungen }\end{array}$ \\
\hline Region & $\begin{array}{l}\text { Charakteristika der Arbeitskräfte, Löhne, Gewerkschaften, Marktzugang } \\
\text { und Dynamik, großräumige Lage, Wirtschaftsstruktur, Lieferanten, } \\
\text { Dienstleistungen, Regionalförderung }\end{array}$ \\
\hline Stadt/Kommune & $\begin{array}{l}\text { Verkehrserschließung (Flug, Bahn, Auto), Quantität und Qualität der } \\
\text { Arbeitskräfte, spezififsche Infrastruktur (Universität, Forschungseinrichtungen), } \\
\text { lokale Wirtschaftspolitik und Förderung, Lebensqualität }\end{array}$ \\
\hline Grundstück & Infrastrukturelle Erschließung, Größe, Preis, Umweltsituation \\
\hline $\begin{array}{l}\text { Quelle: Maier, G./Tö } \\
\text { Standorttheorie und }\end{array}$ & $\begin{array}{l}\text { 2006): Regional- und Stadtökonomie } 1 . \\
\text { tur, Wien, New York, S. } 33 .\end{array}$ \\
\hline
\end{tabular}

das Einkommensteuer- und Rechtssystem, die politische und wirtschaftliche Stabilität, Geldwertstabilität und die Wirtschaftsverfassung. In die zweite Ebene (Region) fallen u. a. das regionale Arbeitsangebot in qualitativer und quantitativer Hinsicht, die vorhandene Infrastruktur in den Bereichen Verkehr, Bildung und Forschung. Zur dritten Ebene (Stadt/ Kommune) zählen unter anderem Grundstückspreise, Umwelt- und Lebensqualität, Markt- und Konkurrenznähe (Übersicht 2).

Die Bedeutung der einzelnen Standortfaktoren variiert von Standort zu Standort. Und natürlich gewichten Unternehmen die jeweiligen Faktoren unterschiedlich, je nach Motiv, Vorhaben, Branche oder Betriebstyp. Das Beschäftigungspotenzial gehört genauso zu den Standortfaktoren wie die Präsenz von Gewerkschaften oder das Lohnniveau.

\section{BESCHÄFTIGTENBILDER}

Die Standortwerbung beschreibt deutsche Beschäftigte durchgängig als , hoch qualifizierte und motivierte Mitarbeiter"; sie seien sehr gut ausgebildet, qualifiziert, hoch motiviert, flexibel und produktiv. Die Beschäftigungsfähigkeit der Arbeitnehmerinnen und Arbeitnehmer werde zudem durch hohe Investitionen in Aus- und Weiterbildung in Deutschland gesichert. Aber dies ist nur eine Seite der Medaille; die andere stilisiert Schwächen der Verhandlungs- und Machtpositionen der Beschäftigten bzw. ihrer Gewerkschaften zu Standortvorteilen. So gilt als eine wesentliche Stärke des Standortes Ostdeutschland, dass die Arbeitsbedingungen im Vergleich zu Westdeutschland - oder sogar im Abgleich zu einzelnen osteuropäischen Ländern schlechter sind. Inhaltlich wird diese Aussage über lange Arbeitszeiten, geringfügige Fehl- bzw. Krankheitszeiten, niedrige Ein- kommen, zurückhaltende Lohnforderungen und die geringe gewerkschaftliche Präsenz vermittelt.

\section{ARBEITSZEITEN}

Vor zwanzig Jahren kämpften die Gewerkschaften vorrangig für die Einführung der 35-Stunden-Woche. In Tarifverhandlungen wurde das Produktivitätswachstum nicht nur in Lohnerhöhungen, sondern auch in Arbeitszeitverkürzungen für die abhängig Beschäftigten umgesetzt. Dieser Prozess ist seit etwa Mitte der 1990er Jahre zum Stillstand gekommen bzw. er wird in ganz Deutschland in Richtung einer erneuten Arbeitszeitverlängerung umgekehrt. Insgesamt liegen die Arbeitszeiten in Ostdeutschland höher als in Westdeutschland. Dieser Tatbestand wird von der Standortwerbung aufgegriffen und als Vorteil für Ostdeutschland verkauft. Arbeitszeiten in Ostdeutschland sind nach der ZukunftsAgentur Brandenburg GmbH, flexibler gestaltbar als in den westlichen Bundesländern " und in vielen europäischen Staaten. ${ }^{2}$ „Thüringer Dienstleistungsunternehmen arbeiten auf Basis der 40-Stunden-Woche. Eine Genehmigung für einen durchgehenden Dreischichtbetrieb an 365 Tagen im Jahr ist für Dienstleister, die ihre Leistungen mittels Telekommunikation erbringen, laut Gesetzestext (...) nicht erforderlich“.3 In zahlreichen Publikationen zur Standortwerbung finden sich beliebig viele weitere Beispiele für die Arbeitszeitflexibilität ostdeutscher Erwerbstätiger: Ein Erwerbstätiger bzw. eine Erwerbstätige in Brandenburg arbeitet „im Jahr über 80 Stunden

2 Kammradt, S./Röser, A. (2008): Der Standort Brandenburg im Vergleich, hrsg. von ZukunftsAgentur (ZAB) Brandenburg $\mathrm{GmbH}$, o. O., S. 8.

3 Landesentwicklungsgesellschaft $\mathrm{mbH}$ Thüringen (LEG) (2005): Call Center in Thüringen, o. O., S. 2. 
mehr als im Bundesdurchschnitt", für $56 \%$ der Betriebe bedeute dies Wochenarbeitszeiten von mehr als 40 Stunden, sodass in keinem anderen Land das Arbeitspensum höher sei als in Brandenburg. ${ }^{4}$ Gegenüber dem deutschen Durchschnitt wird in Sachsen rund 77 Stunden pro Jahr mehr gearbeitet, „denn Sachsen und Sächsinnen gehen gern ,auf Arbeit', wie es hier heißt“. Den über dem deutschen Wert liegenden Überstundenzahlen pro Woche in den MOELändern wird entgegnet, dass diese nur mit „zum Teil erheblich höherer Streiktagezahl“ erkauft werden könne (vgl. Fußnote 4).

\section{ENTLOHNUNG}

Die Erwerbseinkommen der Mehrheit der Beschäftigten in Deutschland werden in Tarifverträgen geregelt und spiegeln ein zentrales Betätigungsfeld der deutschen Gewerkschaften wider. Die Einkommenshöhe ist von vielen Faktoren - Branche, Tätigkeit, Arbeitszeit etc. - abhängig. Auch regional unterscheiden sich die durchschnittlichen Verdienste der abhängig Beschäftigten. Auf Unternehmensseite schlägt die Entlohnung der Beschäftigten als Kostenfaktor zu Buche. Es überrascht deshalb nicht, dass die ostdeutsche Wirtschaftsförderung die in Ostdeutschland im Vergleich zum Westen niedrigeren Einkommen als weiteren Standortvorteil sieht. Doch auch die Lohnstabilität in Deutschland insgesamt wird geschätzt. So schreibt z. B. die Berlin Partner GmbH, dass die deutschen Löhne auf lange Sicht wettbewerbsfähiger seien als die anderer Länder und „damit auch mittelfristig kalkulierbar“. Anders als in den Nachbarstaaten, die in den letzten Jahren deutliche Lohnsteigerungen verbuchten, stiegen die deutschen Löhne nur moderat an. Betrachtet man zudem nur die Bundesländerebene fällt Ostdeutschland aus Sicht der Marketingstrategien deshalb "positiv" auf, weil die Löhne dort sogar noch $33 \%$ niedriger als in Westdeutschland sind. Brandenburg wirbt sogar mit Arbeitskosten, die $38 \%$ unter dem Westniveau liegen (so die ZukunftsAgentur Brandenburg $\mathrm{GmbH}$ ). Berlin wiederum vergleicht seine Löhne mit denen anderer deutscher Großstädte, die um durchschnittlich $20 \%$ höher lägen.

Laut Standortwerbung wirke sich die geringe Lohnentwicklung auf die Lohnstückkosten aus, was potenziellen Investoren einen bedeutenden Vorteil verschaffe.
Deutschlands Lohnstückkosten lagen 2005 $10 \%$ unter dem EU-Durchschnitt (gemessen 2005) und verzeichneten zwischen 1995 und 2004 den geringsten Anstieg in der EU. In Ostdeutschland sanken die Lohnstückkosten im Verarbeitenden Gewerbe sogar kontinuierlich. Nach Angaben der Brandenburgischen Wirtschaftsfördergesellschaft ZukunftsAgentur Brandenburg GmbH im Jahr 2006 um -8,4\%. Brandenburg steht im Wettbewerb der niedrigsten Lohnstückkosten an zweiter Stelle nach Sachsen-Anhalt (Zahlen aus 2004). ${ }^{5}$ „Trotz der überdurchschnittlichen Qualifikation der Mitarbeiter liegen die Löhne und Gehälter in der Region ca. 30 \% unter denen vergleichbarer Standorte. Flexible Arbeitszeiten und Tarife, 40 StundenArbeitswoche sowie wenige Feiertage in der Region sind kostensparend (...)"6

Die einzelnen Bundesländer konkurrieren in der Standortwerbung nicht nur untereinander mit niedrigen Löhnen, Referenzgröße sind auch die neuen EU-Mitglieder Mittel- und Osteuropas. Da werden zum einen die eigenen Arbeitskosten gegenüber den Standorten in Polen und Tschechien schön gerechnet, zum anderen wird die Attraktivität der mittel- und osteuropäischen Staaten schlecht geredet, weil dort die Arbeitskosten schneller gestiegen sind als in Deutschland. Das Credo der Standortwerbung lautet: Standorte in Deutschland seien für Unternehmen mittelfristig besser zu kalkulieren. In den neuen EU-Ländern (Tschechien, Ungarn, Polen, der Slowakei und Slowenien) erfolge ein Aufholprozess, und die Gehaltsstrukturen einiger Bereiche entsprächen schon fast westeuropäischem Niveau. ${ }^{7}$

\section{GERINGE GEWERKSCHAFTLICHE PRÄSENZ}

Auch die Sozialpartnerschaft in Deutschland ist Thema der Standortwerbung. Auf der einen Seite werden die guten Beziehungen zwischen Arbeitgebern und Gewerkschaften als positiver Standortfaktor vermarktet. Verwiesen wird auf die seltenen Auseinandersetzungen zwischen den Tarifparteien bzw. auf die extrem niedrige Anzahl von Streiktagen. Durch Streiks gingen dagegen in Ungarn jahresdurchschnittlich 15,8 Arbeitstage je 1.000 Beschäftigte verloren, in Rumänien 55,8 Tage und in Spanien sowie Dänemark sogar über 140 Tage; Deutschland setzt sich mit 2,4 Streiktagen je 1.000 Beschäftigte im Zeitraum von 1996 bis 2005 deutlich ab (vgl. Fn. 4). Auf der anderen Seite - das gilt für Ostdeutschland - wird auch gerne mit der geringen gewerkschaftlichen Präsenz als positivem Standortfaktor geworben, was dem Bild einer gut funktionierenden Sozialpartnerschaft zuwider läuft. Tarifvertragsfreie Betriebe als Standortvorteil: Damit wirbt beispielsweise das Business Location Center in Berlin-Brandenburg: „Ein Grund für diesen Kostenvorteil ist, dass mehr als $70 \%$ der Brandenburger Unternehmen nicht an Tarifverträge gebunden sind und daher die Löhne mit ihren Mitarbeitern individuell aushandeln können." Auch die Wirtschaftsförderung Sachsen GmbH wirbt mit ganz individuellen Betriebsvereinbarungen, die den Wirtschaftsstandort zur „Bestform“ auflaufen lassen. Und das aus dem einfachen Grund, weil „die Sachsen" eben pragmatisch denken und handeln. „Sie kennen ihren Wert, verlieren aber die Unternehmen nicht aus dem Blick“ (http://www.invest-in-saxony.de/ set/157/Image_Deutsch.pdf).

\section{BRANCHENSPEZIFISCHE STANDORTWERBUNC}

Die Standortwerbung setzt auch auf branchenspezifische Ansprachen, indem sie für spezielle Branchen Werbematerialien vorhält. In diesem Zusammenhang fällt auf, dass sich die Callcenter-Branche aus Beschäftigtensicht am negativsten präsentiert. Als arbeitsintensive Branche, in der Produktivitätszuwächse nur sehr begrenzt durch technische (Produkt- und Prozess-) Innovationen zu erzielen sind, bleiben im Wesentlichen nur noch die Lohn- und Arbeitskosten, wodurch sich die potenziellen Standorte für diese Branche auszeichnen können. In der Tat wird mit den niedrigen, ,äußerst wettbewerbsfähigen Lohnkosten" in Ostdeutschland geworben, so z. B. bei der Standortwerbung der Investitions- und Marketinggesellschaft SachsenAnhalt mbH. Die Callcenter-Branche wirbt bei der Darstellung möglicher Standortvorteile im Vergleich zu anderen Branchen schwerpunktmäßig mit den schlechten Arbeitsbedingungen, sodass die Gewichtung

4 Kammradt, S./Röser, A. (2008): a. a. O., S. 17ff. 5 Kammradt, S./Röser, A. (2008): a. a. O., S. 31.

6 Business Location Center (o. J.): Logistik in der Hauptstadtregion Berlin-Brandenburg, o. O., S. 2. 7 Kammradt, S./Röser, A. (2008): a. a. O., S. $27 f f$. 
niedriger Lohnkosten höher ist als in anderen Branchen des jeweiligen Bundeslandes, für die auch mit niedrigen Lohnkosten geworben wird: so z. B. in Berlin, Brandenburg und Thüringen.

Doch es gibt auch Gegenbeispiele: In Berlin und Brandenburg verzichten die IuK-Industrie, die „Games Industry“ und die neuen Schlüsseltechnologien - Optik, Mikro, Nano - auf das Hohelied unterdurchschnittlicher Löhne. In Thüringen setzen sich die Optische Industrie, Biotechnologie und Medizintechnik von der Methode ab, mit schlechten Arbeitsbedingungen zu werben. Scheinbar gewinnt auch bei den Standortstrategen die Erkenntnis Raum, dass in Unternehmen, insbesondere in den Hightech-Branchen, qualifizierte Beschäftigte benötigt werden, die man nicht mit schlechten Arbeitsbedingungen bekommen wird.

\section{FAZIT}

Nach dem Rückzug Nokias aus Deutschland ging ein Aufschrei durch die Republik. Die öffentliche Empörung und Kritik galt einem Unternehmen, das entfesselte Renditeerwartungen auf dem Rücken seiner Beschäftigten realisieren will. Dass die Öffentlichkeit allmählich sensibler auf derartige Unternehmensstrategien reagiert, stimmt hoffnungsvoll. Unsere Auswertung der Standortwerbung lehrt uns aber auch: Die ostdeutschen Bundesländer wie auch der Bund werben mit schlechten Arbeitsbedingungen für den Wirtschaftsstandort Ostdeutschland. Staatliche bzw. für staatliche Stellen handelnde Institutionen preisen Arbeitsbedingungen und Standards in Ostdeutschland an, die unter dem Westniveau liegen. Sie sollen Anreiz sein, Unternehmen und Investitionen für den Standort Ostdeutschland zu gewinnen. Die Standortprä- sentationen erwecken den Anschein, dass Ostdeutschland nicht Teil einer hoch entwickelten Industrienation sei, in der die Qualität der Arbeitsplätze eine wichtige Rolle spielt, womit sich Deutschland international abhebt und ein Aushängeschild für eine stabile Volkswirtschaft ist, in der alle von dem erwirtschafteten Wohlstand profitieren können. Die Frage steht im Raum: Wird hier nicht unter staatlicher Verantwortung, zumindest Duldung, ein Unternehmens- und Unternehmertyp gefördert, für den soziale bzw. Beschäftigtenverantwortung ein Fremdwort geworden ist? Ein Umdenken in der Standortwerbung ist dringend erforderlich. Ein erster Schritt in die richtige Richtung wäre bereits getan, wenn in naher Zukunft Textpassagen, die mit schlechten Arbeitsbedingungen für den Standort Ostdeutschland werben, aus dem Internet entfernt würden - und dann auch aus den Köpfen verschwänden. 